NEUROLOGICAL RARITIES

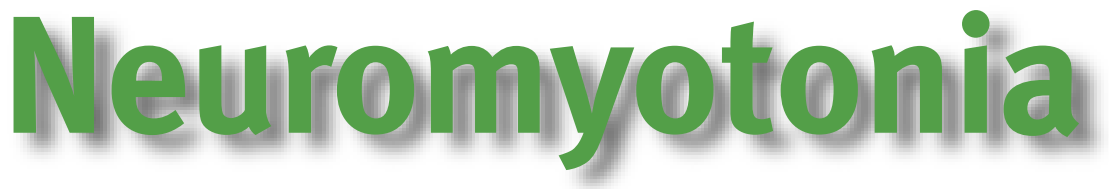

Table 1 Clinical associations of neuromyotonia

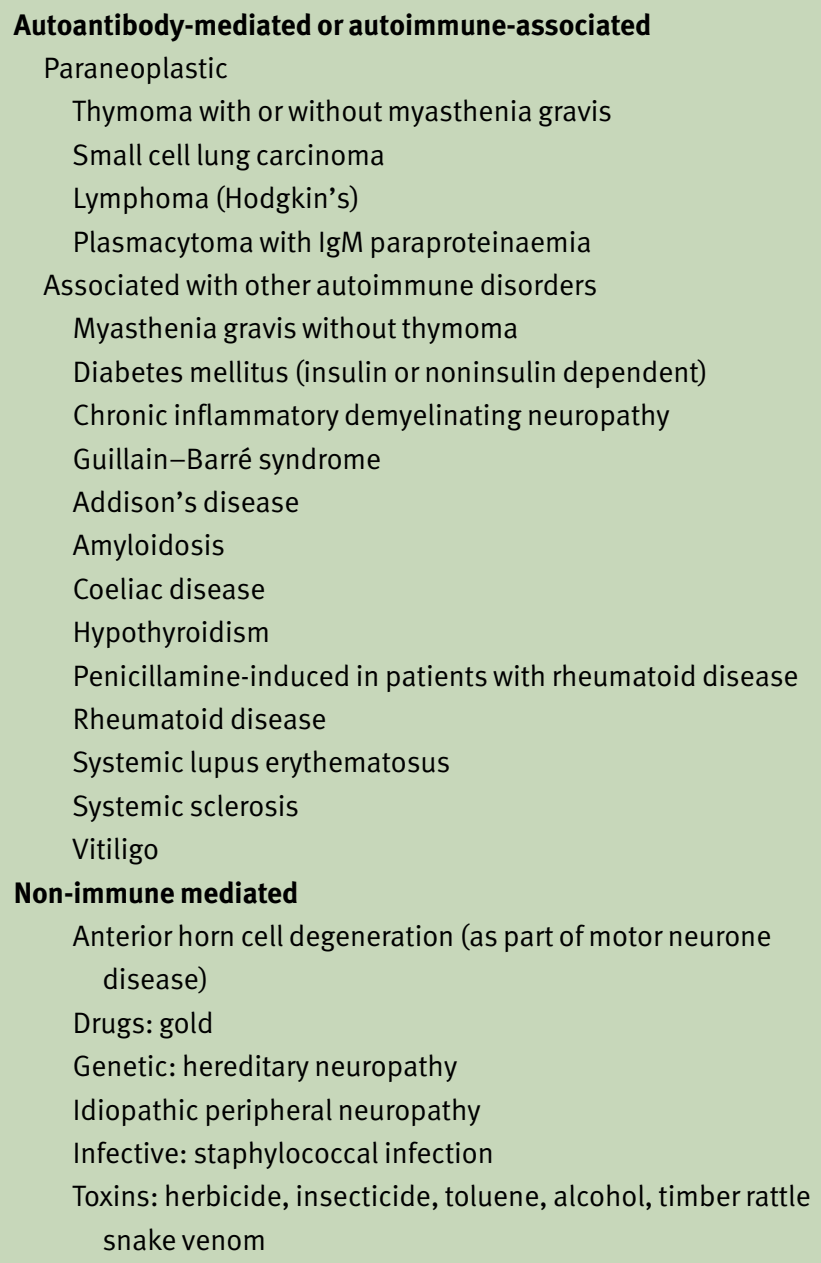

\section{Paul Maddison}

Department of Neurology, Royal Victoria Infirmary, Newcastle upon Tyne, UK; Email: paul@piglet2.demon.co.uk

Practical Neurology, 2002, 2, 225-229

\section{INTRODUCTION}

Neuromyotonia is a very rare condition of spontaneous, continuous muscle activity of peripheral nerve origin. It is characterized clinically by muscle twitching at rest (visible myokymia), cramps that can be triggered by voluntary or induced muscle contraction, and impaired muscle relaxation (pseudomyotonia). Often, patients also have symptoms of excessive sweating and more rarely mild muscular weakness, and paraesthesia Following the electrophysiological account in Denny-Brown \& Foley 1948 of 'undulating myokymia', the first full description of the syndrome of 'continuous musclefibre activity' was made by Isaacs (1961). He established the peripheral nerve origin of the spontaneously-occurring discharges by noting the persistence of abnormal EMG activity after proximal nerve block, no change in spontaneous muscle activity during general anaesthesia with thiopentone, and that the depolarizing muscle relaxant succinylcholine and neuromuscular blocking agent curare both produce electrical silence after a few minutes.

\section{PATHOPHYSIOLOGY}

Neuromyotonia can sometimes be seen in isolation, or as a feature of genetically inherited diseases such as Episodic Ataxia Type I, or Hereditary Motor and Sensory Neuropathy. But most commonly, it is associated with other disorders and in many cases there seems to be an autoimmune association (Table 1).

\section{Autoimmune aetiology}

The association of neuromyotonia with thymoma, myasthenia gravis, vitiligo, Hashimoto's thyroiditis and penicillamine treatment provid- 
ed some clinical evidence of a possible autoimmune aetiology. Spontaneous remission has also been observed, a finding consistent with autoimmunity. In addition, neuromyotonia has been reported in patients with lung cancer, raising the possibility that tumour antigenic determinants are perhaps capable of triggering an autoimmune response producing antibodies that cross-react with neuronal voltage-gated ion channels.

The first direct evidence of an autoimmune aetiology came from the demonstration of a significant reduction in the number of neuromyotonic discharges recorded by EMG after plasma exchange (Sinha et al. 1991). In addition, purified IgG from the same patient, when injected intraperitoneally into mice, enhanced in vitro resistance to d-tubocurarine at the neuromuscular junction of phrenic nervehemidiaphragm preparations. Further studies showed significantly-increased acetylcholine quantal content at the neuromuscular junction in similarly treated mice, mimicking the $\mathrm{K}^{+}$ channel blocking effect of 3,4-diaminopyridine (Shillito et al. 1995). Therefore, it was proposed that the increase in nerve terminal excitability could be due to interference with the function of neuronal $\mathrm{K}^{+}$channels that ordinarily stabilize membrane potential. Several other patients were subsequently reported as showing an improvement in symptoms and signs following plasma exchange, confirming the presence of a pathogenic circulating factor (Newsom-Davis \& Mills 1993).

\section{Anti-voltage-gated potassium channel antibodies and autoim- mune neuromyotonia}

The discovery of an autoimmune basis for neuromyotonia, coupled with the finding of increased nerve terminal excitability, led to the supposition that voltage-gated potassium channels (VGKCs) were the primary target for the pathogenic antibodies (Sinha et al. 1991). The phenotypic and electrophysiological similarities between the Shaker mutant of the fruit fly Drosophilia melanogaster and patients with neuromyotonia further suggested that VGKCs were functionally blocked by antibodies. However, it wasn't until the neurotoxin dendrotoxin was first used in radiolabelled form in an immunoprecipitation assay that the nature of the putative antibody was identified.

Dendrotoxin is an eastern green mamba snake (Dendroaspis, Fig. 1) venom peptide that is capable of occluding the pore of VGKCs. Dendrotoxin homologueues specifically bind to and block some isoforms of VGKCs. A radioimmunoassay using ${ }^{125} \mathrm{I}$ - -dendrotoxin-labelled extracts of human frontal cortex was developed that detected anti-VGKCantibodies in abouthalf the patients with acquired autoimmune neuromyotonia (Shillito et al. 1995). However, as dendrotoxin is not capable of blocking all members of the Shaker-related VGKC family, a more sensitive molecular immunohistochemical assay was developed that showed VGKC antibody binding in every one of 12 neuromyotonia patients but not in any controls (Hart et al. 1997).

Figure 1 Dendroaspis, the green mamba snake.

(C) Martin Overton (arachnophiliac.com)

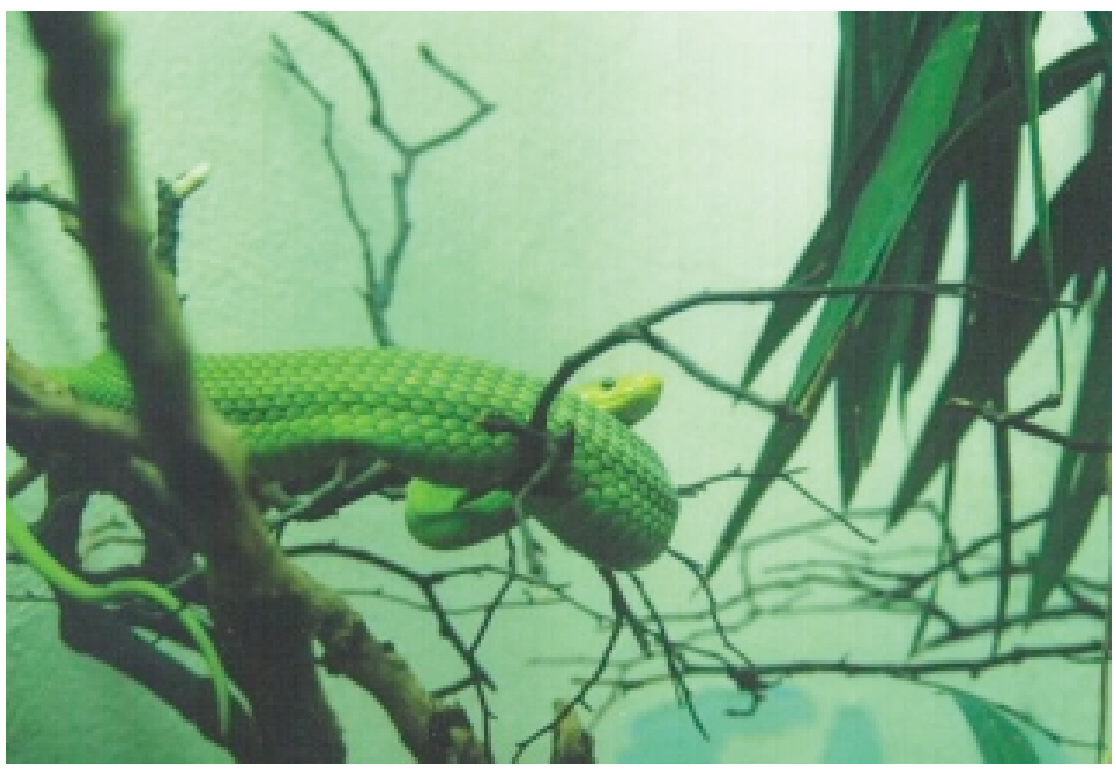




\section{Muscle twitching or 'visible}

\section{myokymia' is observed as}

\author{
a continuous, undulating,
} wave-like rippling of muscles,

\section{likened to a bag of worms}

\section{under the skin; muscle rippling}

\section{can sometimes be felt on}

\section{palpation, even when it is \\ invisible to the naked eye}

\section{CLINICAL DESCRIPTION}

Neuromyotonia appears sporadically and can occur at any age. It has even been reported in newborn babies of mothers without the condition; these babies all died of respiratory complications in infancy.

\section{Muscle twitching}

Muscle twitching or 'visible myokymia' is observed as a continuous, undulating, wave-like rippling of muscles, likened to a bag of worms under the skin. This is usually the commonest symptom, seen in over $90 \%$ of patients. Twitching generally occurs in the limbs but can also be seen in the trunk muscles and the face, including the tongue. Rarely, the laryngeal muscles are involved, causing hoarseness and exertional dyspnoea. Occasionally, there is no visible muscle twitching at all, but needle EMG reveals continuous motor unit activity. However, muscle rippling can sometimes be felt on palpation, even when it is invisible to the naked eye.

\section{Cramps}

Muscle cramps that can be painful at times are a prominent feature in over $70 \%$ of cases and are sometimes the first symptom that is noticed. They may be associated with spasms and are sometimes worsened by attempted voluntary muscle contraction or electrical nerve stimulation. Cold weather can also precipitate muscle cramps.

\section{Muscle stiffness}

This can occur in association with cramps, and can be severe enough to affect walking and man- ual dexterity. As a result, patients may adopt an abnormal posture and may be unable to stand on their heels due to stiffness in the lower limbs. Stiffness can also occur in the muscles of respiration resulting in breathlessness. Occasionally, stiffness can improve with repeated exercise. It is sometimes impossible to elicit the tendon reflexes due to the increased muscle tone.

\section{Increased sweating}

Hyperhidrosis, seen in about half the cases, is a systemic feature that is thought to arise as a result of an increase in the basal metabolic rate, perhaps due to continuous muscle activity. There is no evidence of continuous neural activity in the sudomotor nerves.

\section{Muscle hypertrophy}

Likehyperhidrosis, musclehypertrophy is thought to arise as a result of continuous muscle activity. Most often the calves are hypertrophied but hypertrophy can also be seen in the forearm and hand muscles. The degree of hypertrophy seems to correlate with the severity of overactivity in individual muscle groups and is usually bilateral.

\section{Pseudomyotonia}

This term describes a myotonic-like slow relaxation of muscles after voluntary contraction, for example abnormally slow release of hand grip. Unlike myotonic dystrophy, there is no evidence of percussion myotonia. Pseudomyotonia may be the first symptom and can occur on eye and jaw closure as well as on hand grip. Only about one third of patients exhibit this phenomenon.

\section{Muscle weakness}

Reduced muscle power is unusual, but has been reported with no additional cause for weakness, even when the weak muscles were hypertrophied. One cause for the muscular weakness may be fatigue in the presence of continuous muscle fibre activity.

\section{Central nervous system symptoms}

There are occasional reports of hallucinations, delusional episodes and insomnia, sometimes referred to as Morvan's fibrillary chorea (Liguori et al. 2001). Although oligoclonal bands have been found in the cerebrospinal fluid (CSF) of patients with autoimmune neuromyotonia, there is no correlation with disease severity or central symptoms. Detectable levels of antivoltage-gated potassium channel antibodies in the CSF have not been demonstrated. 


\section{EMG reveals DIAGNOSIS}

\section{spontaneous, Electromyographic features}

EMG reveals spontaneous, continuous, ir regularly occurring doublet, triplet or multiplet single motor unit discharges, firing at a high intraburst frequency $(30-300 \mathrm{~Hz})$ (Fig. 2). In addition, fibrillation potentials and fasciculations are often present, the former indicating the discharge of single muscle fibres. The shape or size of the motor unit during a train of discharges can vary, due to the depolarization of different muscle fibres supplied by the same motor nerve (Fig. 3). Several different motor units may be seen to be discharging in the same EMG recording. Most spontaneous discharges occur in distal muscles and usually no more than

\section{at a high intraburst}

frequency

Figure 2 A 25 s continuous needle EMG recording from medial gastrocnemius.

Motor units are seen to fire spontaneously and irregularly as doublets (B), triplets (A), and multiplets (C), with intraburst frequencies of up to $120 \mathrm{~Hz}$. The prolonged discharge in the middle of the recording consists of rapidly firing triplets of more than one motor unit (D).

Figure 3 Sections from longer continuous EMG recordings illustrating decremental patterns occurring within high frequency bursts of neuromyotonia.
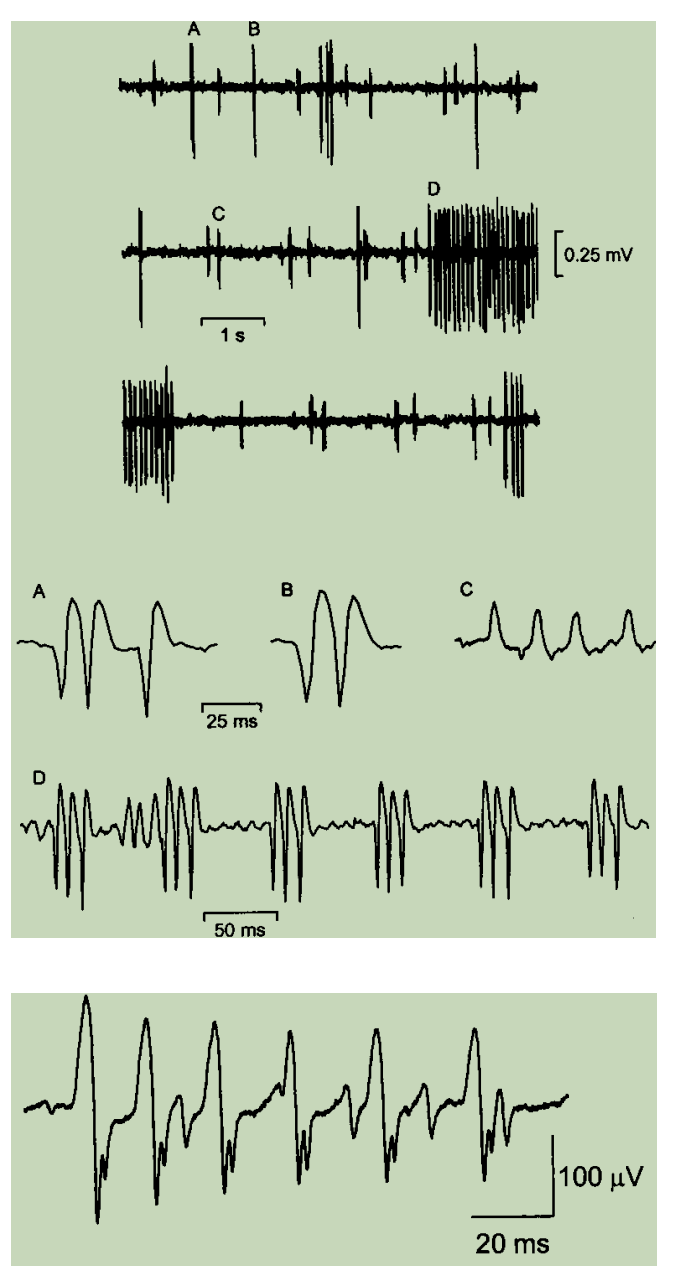

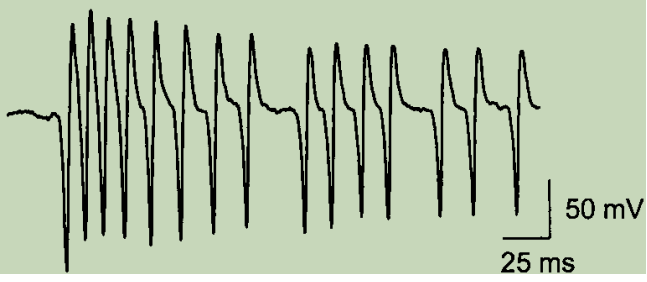

10 different motor unit (or partial motor unit) discharges are seen (Hart et al. 2002).

Characteristically, electrical stimulation of the nerve results in increased spontaneous activity seen as after-discharges (Fig. 4), and voluntary muscle contraction can provoke spontaneous motor unit activity lasting several minutes. Neuromyotonic discharges are characteristically present during sleep.

Using electrophysiological techniques, it has been possible to determine the site of origin of the spontaneous electrical activity. Regional curarization abolishes the discharges, whereas general anaesthesia does not. This suggests that they arise from peripheral nerve. Several accounts have shown a reduction in the number of discharges following local anaesthetic nerve block at the elbow or knee, indicating that the ectopic foci lie in the proximal segments of the nerve and its root. However, other reports show no diminution of spontaneous electrical activity after proximal nerve block, even when blocked at the wrist, suggesting a more distal discharge generation site. It is most probable that the generator sites lie anywhere along the whole length of the nerve, from the root to the terminal arborizations.

Other patients with milder symptoms of muscle cramps, twitching, and stiffness, who share similar autoimmune associations as autoimmune neuromyotonia, have EMG features similar to the cramp-fasciculation syndrome, but without the doublet, triplet, or multiplet motor unit discharges usually seen in neuromyotonia. These EMG features reflect quantitative rather than qualitative differences between the diverse clinical syndromes of cramp-fasciculation syndrome at one end, and acquired neuromyotonia at the other, more severe end of a spectrum of autoimmune peripheral nerve hyperexcitability (Hart et al. 2002).

\section{Immunological findings}

Immunoprecipitation assays detect anti-VGKC antibodies in the serum of about $40 \%$ of patients with acquired neuromyotonia, in $80 \%$ if there is an associated thymoma. Associated autoimmune disorders and other autoantibodies can be detected in approximately $50 \%$ of neuromyotonia patients, most notably antiacetylcholine receptor antibodies, indicating coexistent myasthenia gravis in about $20 \%$.

\section{DIFFERENTIAL DIAGNOSIS}

Autoimmune neuromyotonia probably represents the severe end of the clinical and elec- 


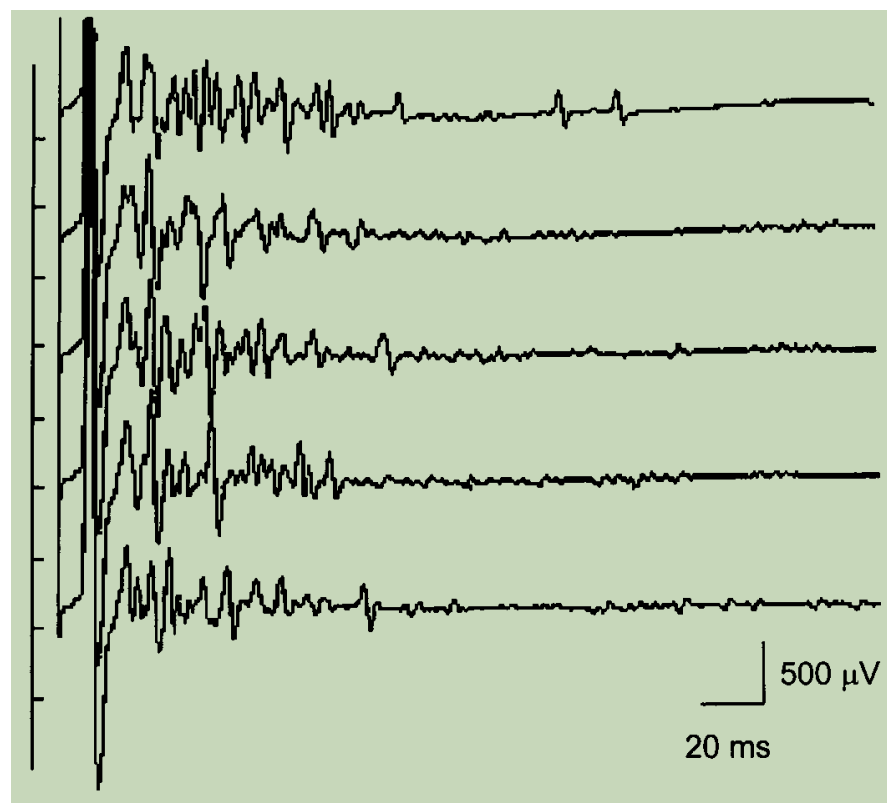

Figure 4 Following supramaximal electrical stimulation of the posterior tibial nerve, five consecutive compound muscle action potentials are followed directly by trains of asynchronous after-discharges. with voltage-gated sodium channels. Patients with acquired neuromyotonia may require immunosuppression in the form of prednisolone and azathioprine, although not all respond fully. Severe symptoms may be ameliorated for up to 4 weeks following plasma exchange. In my experience, quantitative clinical and electrophysiological responses to intravenous immunoglobulin therapy are disappointing, although there have been no randomised controlled trials of this, or of any other treatments. The association of neuromyotonia with thymoma, smallcell lung cancer and lymphoma necessitates the search for, and subsequent treatment of these malignancies. However, the treatment of the tumour often has little effect on the clinical severity of the peripheral nerve hyperexcitability.

\section{THE MAIN CLINICAL FEATURES OF NEUROMYOTONIA \\ - Muscle twitching \\ - Muscle cramps \\ - Muscle stiffness \\ - Muscle hypertrophy \\ - Pseudomyotonia \\ - Muscle weakness rarely \\ - Increased sweating} if the peripheral nerve hyperexcitability, or 'visible myokymia' is clinically and electrophysiologically well localized (Maddison et al. 2000). The motor unit discharge rate in facial or limb myokymia is generally slower than neuromyotonia, usually not exceeding $60 \mathrm{~Hz}$.

The stiff-man syndrome is a similar condition of continuous motor unit activity, where abnormal excitability of spinal interneuronal networks and descending control over the anterior horn cell is thought to be the underlying pathophysiology. Unlike neuromyotonia, axial rigidity is a prominent feature and patients have a characteristic gait, with marked hyperlordosis. Autoantibodies to glutamic acid decarboxylase are present in about $40 \%$ of these patients. The central rather than peripheral origin of the excess motor unit activity in stiff-man syndrome, in contrast to neuromyotonia, is confirmed by the disappearance of discharges during sleep, general anaesthesia and peripheral nerve block.

\section{TREATMENT}

The symptoms of peripheral nerve hyperexcitability often respond well to anticonvulsants such as phenytoin, carbamazepine, sodium valproate and lamotrigine, all of which primarily reduce neuronal repetitive firing through interaction 